\title{
PSYCHOLOGICAL STRESS AND RECURRENT APHTHOUS STOMATITIS
}

\author{
Camila de Barros Gallo, ${ }^{\mathrm{I}}$ Maria Angela Martins Mimura, ${ }^{\mathrm{II}}$ Norberto Nobuo Sugaya ${ }^{\mathrm{I}}$
}

doi: 10.1590/S1807-59322009000700007

Gallo CB, Mimura MAM, Sugaya NN. Psychological stress and recurrent aphthous stomatitis. Clinics. 2009;64(7): 645-8.

INTRODUCTION AND OBJECTIVES: Recurrent aphthous stomatitis (RAS) is the most common type of ulcerative disease of the oral mucosa. Despite its worldwide occurrence and the extensive amount of research that has been devoted to the subject, the etiology of RAS remains unclear. Nevertheless, several hereditary, nutritional, infectious and psychological factors have been associated with RAS. The aim of this case-control study was to assess the influence of psychological stress on the manifestation of RAS. METHOD: Fifty patients were enrolled in the trial. Twenty-five RAS patients constituted the study group and another 25 nonRAS patients who were similarly matched for sex, age and socioeconomic status constituted the control group. Each patient was evaluated in terms of the four domains of stress (emotional, physical, social and cognitive) using an internationally validated questionnaire, which was comprised of 59 items and measured the frequency and intensity of stress symptoms. The RAS group was interviewed during an active RAS episode. Completed questionnaires were submitted to proper analytical software and interpreted by an expert psychologist.

RESULTS: There was a higher level of psychological stress among RAS group patients when compared to the control group $(P$ $<0.05)$.

CONCLUSION: Psychological stress may play a role in the manifestation of RAS; it may serve as a trigger or a modifying factor rather than being a cause of the disease.

KEYWORDS: Aphthous stomatitis; Psychological stress; Canker sores; Ulcerative disease.

\section{INTRODUCTION}

Recurrent aphthous stomatitis (RAS) is the most common type of ulcerative disease of the oral mucosa, and it affects approximately $20 \%$ of the general population. The classic presentation of RAS is recurrent, self-limiting ulcers that mainly affect nonkeratinized oral mucosa. A prodromal burning sensation lasting 24 to 48 hours can often precede the onset of ulcers. Minor RAS, which makes up more than $80 \%$ of all RAS cases, is a small (up to $1 \mathrm{~cm}$ in diameter), shallow, painful, well-circumscribed, and round-shaped ulceration that is covered by a yellow-grayish

'Disciplina de Estomatologia Clínica, Departamento de Estomatologia, Faculdade de Odontologia da Universidade de São Paulo - São Paulo/SP, Brazil. "I Disciplina de Semiologia, Faculdade de Odontologia da Universidade Ibirapuera - São Paulo/SP, Brazil

Email: camilagallo@gmail.com

Tel / Fax: 55113091.7883

Received for publication on February 02, 2009

Accepted for publication on April 15, 2009 pseudomembrane and surrounded by an erythematous halo. The ulceration generally heals without scarring after 10 to 14 days. Major RAS is characterized by ulcers that are typically larger and deeper than minor RAS. Furthermore, they heal more slowly and often cause scarring. Herpetiform ulcers manifest as multiple recurrent clusters of small ulcers (less than $4 \mathrm{~mm}$ in diameter) that are scattered throughout the oral mucosa. These ulcers may further coalesce into larger ulcerations..$^{1-5}$

The worldwide distribution, high frequency and decreased quality of life generated by RAS have resulted in a great deal of research into the etiology and efficient therapy of this disease. However, the etiology of RAS still remains unclear, and the currently available therapy remains inadequate. On the other hand, many factors have already been implicated in the promotion and/or exacerbation of RAS; these include positive family history, local trauma, nutritional deficiency, food hypersensitivity, immune disturbance, smoking cessation, and psychological stress, among others. ${ }^{1-5}$ 
Previous studies have suggested that psychological disturbances such as stress and anxiety could play a role in the onset and recurrence of RAS lesions. However, the obtained results were rather varied. ${ }^{6-12}$ Therefore, the aim of this study was to conduct a case-control investigation of the influence of psychological stress on RAS onset. This was done using an internationally validated questionnaire ${ }^{13}$ that evaluated the patients' level of stress by assessing the presence, frequency and intensity of stress symptoms.

\section{MATERIALS AND METHODS}

The trial was instituted during a period of one year at the Dentistry School of Sao Paulo University after approval by the local Ethics Committee.

Two groups of patients were recruited after giving informed consent: one group was composed of RAS patients (RAS group) and the other was composed of sex-, age- and socioeconomically-matched non-RAS patients (control group). The socioeconomic status was determined using a printed chart developed by Graciano et al. ${ }^{14}$

The inclusion criteria for RAS patients were as follows: minor form of RAS, non-smokers, and a minimum of two years of RAS history. RAS diagnosis was established on a clinical basis, and patients were classified according to the type of ulcers and the severity of RAS episodes. The control group consisted of patients who were seeking dental treatment at the Dentistry School, who had no history of RAS and who were matched to the variables presented by the RAS patient group. Patients with systemic illnesses such as Behçet Syndrome, Celiac or Crohn's disease were excluded.

Clinical severity was classified according to the following parameters: 1 . Mild - less than 6 episodes of RAS per year, one to two lesions per episode; 2 . Moderate between 6 to 12 RAS episodes per year, one to two lesions per episode; 3. Severe - more than 12 RAS episodes per year, multiple ulcerations per episode, or no ulcer-free episodes. ${ }^{15}$

Psychological stress was assessed through a questionnaire developed by the Psychology Institute of Sao Paulo University (Symptoms of Stress List; SSLVAS questionnaire). ${ }^{13}$ Assessment was performed by a single researcher for all patients. The questionnaire was given to patients with active episodes of RAS, and to control patients with appointments for dental treatment. The questionnaire was composed of 59 questions that investigated the frequency and intensity of stress symptoms in emotional, physical, social and cognitive categories of psychological stress. Results were expressed as a score of overall stress level after proper software analysis by an expert psychologist.

\section{RESULTS}

Fifty patients were enrolled in this trial. The RAS group comprised 17 women and 8 men, and the mean age was 34.4 years (range 19-58 yr). Three patients presented with mild RAS, 11 presented with moderate symptoms and 11 presented with severe RAS. The control group was comprised of the same number of patients with the same gender ratio as the RAS group. The mean age of patients in the control group was 34.5 years (range 18-55 yr). Socioeconomic status was similarly distributed throughout the three socioeconomic classes ( 8 lower, 11 medium, 6 high).

All patients indicated a modifying factor that was associated with their lesions, particularly recent exposures to acidic food or local trauma. Seventeen patients reported a relationship between the severity of RAS episodes and stressful life events. Additionally, 16 patients reported that the lesions themselves increased their stress levels.

Completed questionnaires were submitted to analysis by an expert psychologist who, with the aid of proper computer software, generated results that were distributed across the four domains of stress in addition to the overall score, which indicates the general level of stress (Table 1). The stress domain that was the most affected in both groups was the emotional domain, followed by cognitive, social and physical stress domains. The acquired results were subjected to statistical analysis (Mann-Whitney test), which showed a significant difference in terms of general stress levels between the RAS and control groups $(P<0.05)$. Within the RAS group, no difference in stress level was observed among mild, moderate and severe RAS patients $(P=0.57)$. Furthermore, no statistical difference was observed between male and female RAS patients $(\mathrm{P}=0.98)$. The Fisher's exact test indicated no statistical difference when comparing patients and controls in terms of stress levels 2 and $3(P=$ 0.55). The same test showed statistical significance when RAS patients and control patients were compared at stress levels above $3(P=0.017)$.

\section{DISCUSSION}

The impact of oral disorders on quality of life has been increasingly recognized as an important outcome measure for clinical trials, ${ }^{16}$ especially since oral disorders frequently have detrimental effects on speech, nutrition, physical appearance, self-esteem and social interaction. RAS frequently affects patient quality of life as a result of long lasting and recurrent episodes of burning pain. ${ }^{11}$

The results of this trial showed that RAS patients exhibited higher stress levels than the control group during 
Table 1 - Distribution of patients and controls according to general stress level and RAS severity

\begin{tabular}{|c|c|c|c|c|c|}
\hline Stress level & 1 & 2 & 3 & 4 & 5 \\
\hline Mild RAS & - & 1 & 1 & 1 & - \\
\hline Mild controls & - & - & 2 & 1 & - \\
\hline Moderate RAS & - & - & 6 & 4 & 1 \\
\hline Moderate controls & - & 1 & 9 & 1 & - \\
\hline Severe RAS & - & 2 & 3 & 4 & 2 \\
\hline Severe controls & - & 3 & 6 & 1 & 1 \\
\hline Subtotal 1 (RAS) & - & 3 & $10 *$ & $9 *$ & $3 *$ \\
\hline Subtotal 2 (controls) & - & 4 & $17 *$ & $3 *$ & $1^{*}$ \\
\hline Total & - & 7 & 27 & 12 & 4 \\
\hline
\end{tabular}

Interpretation of general level of stress' scores (stress phases): 1- low (low risk phase), 2- medium-low (alert phase), 3- medium (resistance phase), 4medium-high (acute danger phase), 5- high (exhaustion phase).

* Statistically significant difference (Exact Fisher test)

their active episodes. The figures in Table 1 show that $12 / 25$ RAS patients exhibited stress levels of 4-5 while only $4 / 25$ of the control patients exhibited the same stress levels. Additionally, most patients in the RAS group indicated a positive relationship between stress and ulcerative episodes. Psychological stress as a triggering factor for RAS has already been mentioned in the literature, and is typically observed during stressful situations ${ }^{12}$ such as school exam periods, dental treatments, ${ }^{7}$ and periods of significant changes in life. ${ }^{17}$ Sixty-eight percent of patients reported that the occurrence of RAS was associated with some of the aforementioned situations, particularly changes in life such as family problems, new job, new position at the job or new location of residence.

Psychological stress induces immunoregulatory activity by increasing the number of leukocytes at sites of inflammation; $;^{18}$ this is a characteristic often observed during the pathogenesis of RAS. ${ }^{5}$ The pathophysiologic consequences of stress on patients are not uniform, especially given the dynamic and complex mechanisms that affect individuals in different ways. Similarly, the same patient may exhibit different degrees of manifestation associated with the same kind of emotional stress. ${ }^{11}$ As the stress level was investigated during active RAS episodes in this trial, the extent to which recurrent ulcers are themselves stressful to patients who suffer from life-long RAS must be considered. Pedersen ${ }^{8}$ evaluated the psychological stress of 22 RAS patients during active and inactive stages of the disease, and despite the patients' claims of positive associations between stress and RAS episodes, there were no differences between active and inactive stages.

The results of this trial suggest an influence of stress on RAS expression, although the trial did not clarify the mechanisms involved. Some investigators have speculated that anxiety could lead to parafunctional oral habits, including lip and cheek biting, and that those physical traumas may initiate the ulcerative process in susceptible individuals. RAS patients enrolled in our trial denied such habits and showed no clinical signs of chronic trauma. Additionally, it is known that minor physical injuries to the oral mucosa in susceptible RAS patients can frequently trigger RAS lesions. ${ }^{10}$

RAS manifestations appear to be influenced by acute psychological stress. Thus, stress-management interventions like relaxation may be beneficial in reducing RAS recurrence. ${ }^{9,11}$

\section{CONCLUSION}

The statistically different levels of psychological stress observed between the RAS and control group support the role of stress as a modifier of RAS. The results also encourage future studies to assess the impact of psychotherapy on RAS onset and symptoms. The lack of a direct correlation between the level of stress and severity of RAS episodes suggests that psychological stress may act as a trigger or modifying factor rather than an etiological factor in susceptible RAS patients.

\section{ACKNOWLEDGMENTS}

The authors thank the Sao Paulo State Research Foundation (FAPESP) for financial support and Esdras Guerreiro Vasconcellos, professor of the Psychology Institute of the University of Sao Paulo for help and support. 


\section{REFERENCES}

1. Vincent SD, Lilly GE. Clinical, historic and therapeutic features of aphthous stomatitis. Oral Surg Oral Med Oral Pathol. 1992;74:79-86.

2. Woo SB, Sonis ST. Recurrent aphthous ulcers: a review of diagnosis and treatment. J Am Dent Assoc. 1996;127:1202-13.

3. Ship JA, Chavez EM, Doerr PA, Henson BS. Recurrent aphthous stomatitis. Quintessence Int. 2000;31:95-112.

4. Casiglia JM. Recurrent aphthous stomatitis: etiology, diagnosis, and treatment. Gen Dent 2002;50:157-66.

5. Scully C, Gorsky M, Lozada-Nur F. The diagnosis and management of recurrent aphthous stomatitis: a consensus approach. J Am Dent Assoc. 2003;134:200-7.

6. Larato DC. Stress and aphthous ulcers. J Acad Gen Dent. 1972;20:25-6.

7. Kaufman AY. Aphthous stomatitis as a featuring syndrome of emotional stress in dental treatment. Quintessence Int Dent Dig. 1976;7:75-8.

8. Pedersen A. Psychologic stress and recurrent aphthous ulceration. J Oral Pathol Med. 1989;18:119-22.

9. Andrews VH, Hall HR. The effects of relaxation/imagery training on recurrent aphthous stomatits: a preliminary study. Psychosom Med. 1990;52:526-35.

10. McCartan BE, Lamey PJ, Wallace AM. Salivary cortisol and anxiety in recurrent aphthous stomatitis. J Oral Pathol Med. 1996;25:357-9.

11. Chiappelli F, Cajulis,OS. Psychobiologic views on stress-related oral ulcers. Quintessence Int. 2004;35:223-7.
12. Soto-Araya M, Rojas-Alcayaga G, Esguep A. Association between psychological disorders and the presence of Oral lichen planus, Burning mouth syndrome and recurrent aphthous stomatitis. Med Oral. 2004;9:17 .

13. Ferreira EAG, Marques AP, Matsutani LA, Vasconcellos EG, Mendonça LLF. Assessment of pain and stress in fibromyalgia patients. Rev Bras Reumatol. 2002;42:104-10.

14. Lehfeld NAS, Graciano MIG, Neves Filho A. Critérios de avaliação para classificação socioeconômica: elementos de atualização - Parte II. Servico Social \& Realidade. 1996;5:171-201.

15. Ship II. Epidemiologic aspects of recurrent aphthous ulcerations. Oral Surg Oral Med Oral Pathol. 1972;33:400-6.

16. Mumcu G, Hayran O, Ozalp DO, Inanc N, Yavuz S, Ergun T, et al. The assessment of oral health-related quality of life by factor analysis in patients with Behcet's disease and recurrent aphthous stomatitis. J Oral Pathol Med. 2007;36:147-52.

17. Sircus W, Church R, Kelleher J. Recurrent aphthous ulceration of the mouth; a study of the natural history,aetiology and treatment. Q J Med. 1957;26:235-49.

18. Redwine L, Snow S, Mills P, Irwin M. Acute psychological stress: effects on chemotaxis and cellular adhesion molecule expression. Psychosom Med. 2003;65:598-603. 